\title{
Prenatal screening for congenital toxoplasmosis
}

Toxoplasma gondii is a parasitic protozoan and is mainly transmitted to humans by eating undercooked contaminated meat or during clearing cat litters. Acute T. gondii infection causes mild symptoms, such as fever, fatigue, and lymphadenopathy in immunocompetent pregnant women, or may be completely asymptomatic. Women infected by this agent prior to conception rarely transmit the infection to the fetus. However, mothers infected acutely during the pregnancy are likely to transmit the protozoan through the placenta to the fetus, with an overall risk of $20-50 \%$. The transmission risk is higher when the mother becomes infected during the third trimester (transmission rate $60-90 \%$ ) in comparison to infections occurring during the first trimester (transmission rate 10 25\%); but, similar to most other TORCH agents, early infections usually result in a poorer fetal outcome, with the majority of first-trimester infections leading to miscarriage. The survivors of prenatally acquired toxoplasmosis during the second trimester present with major brain abnormalities and severe chorioretinitis; while infections acquired during the third trimester present with less severe findings, such as brain calcifications, mild chorioretinitis, and hepatosplenomegaly.

The principal fetal abnormalities in congenital toxoplasmosis (CTX) are classically described as the triad of chorioretinitis, intracranial calcifications, and hydrocephalus. However, it is estimated that only 1 out of 6 infected fetuses shows two or three findings of this classic triad. About $80 \%$ of infants are asymptomatic at birth, but they may present the complications during late childhood or adolescence. Therefore, making an early correct diagnosis and initiating the appropriate treatment is of paramount importance. In the regions of active parasitic transmission, maternal serologic screening should be offered to all pregnant women, and diagnostic fetal testing (i.e. amniotic fluid polymerase chain reaction) should be suggested to the confirmed cases of recent acute infection (Fig. 1). ${ }^{[1-4]}$

In developing countries, however, serologic screening of all pregnant women may not be possible, but upon prenatal ultrasound findings, one may suspect CTX and initiate appropriate anti-protozoa treatment after confirming the diagnosis by serologic and molecular testing on the maternal serum and amniotic fluid.

The main fetal neurosonographic findings suggestive of CTX include parenchymal calcifications and hydrocephalus (Fig. 2A). These findings may be appreciated in the affected fetuses from 18 - 22 weeks' gestation onwards (depending on the time of infection). Parenchymal calcifications in CTX are seen as foci of hyperechogenicity, with or without posterior shadowing, that are randomly distributed throughout the brain. This finding has been described as a fairly characteristic feature for CTX compared with the mostly periventricular calcifications in congenital cytomegalovirus (CMV) and Zika virus (ZIKV) infections. Microcephaly, i.e. a head circumference that measures 2 standard deviations below the mean for the gestational age, is another possible finding but its prevalence in CTX is much less than in congenital CMV and ZIKV infections. Malformations of cortical development (such as underopercularisation), as well as dysplasia of the corpus callosum, which are commonly seen in congenital ZIKV infection and with a lower prevalence in congenital CMV infection, have been rarely described in CTX. Parenchymal cystic changes secondary to abscess formation is another uncommon feature in CTX.

Non-neurologic findings (Figs 2B and 2C) such as hepatosplenomegaly, intrahepatic echogenicities, peritoneal calcification, and evidences of hydrops fetalis (e.g. ascites, pleural effusion, and pericardial effusion), as well as placental

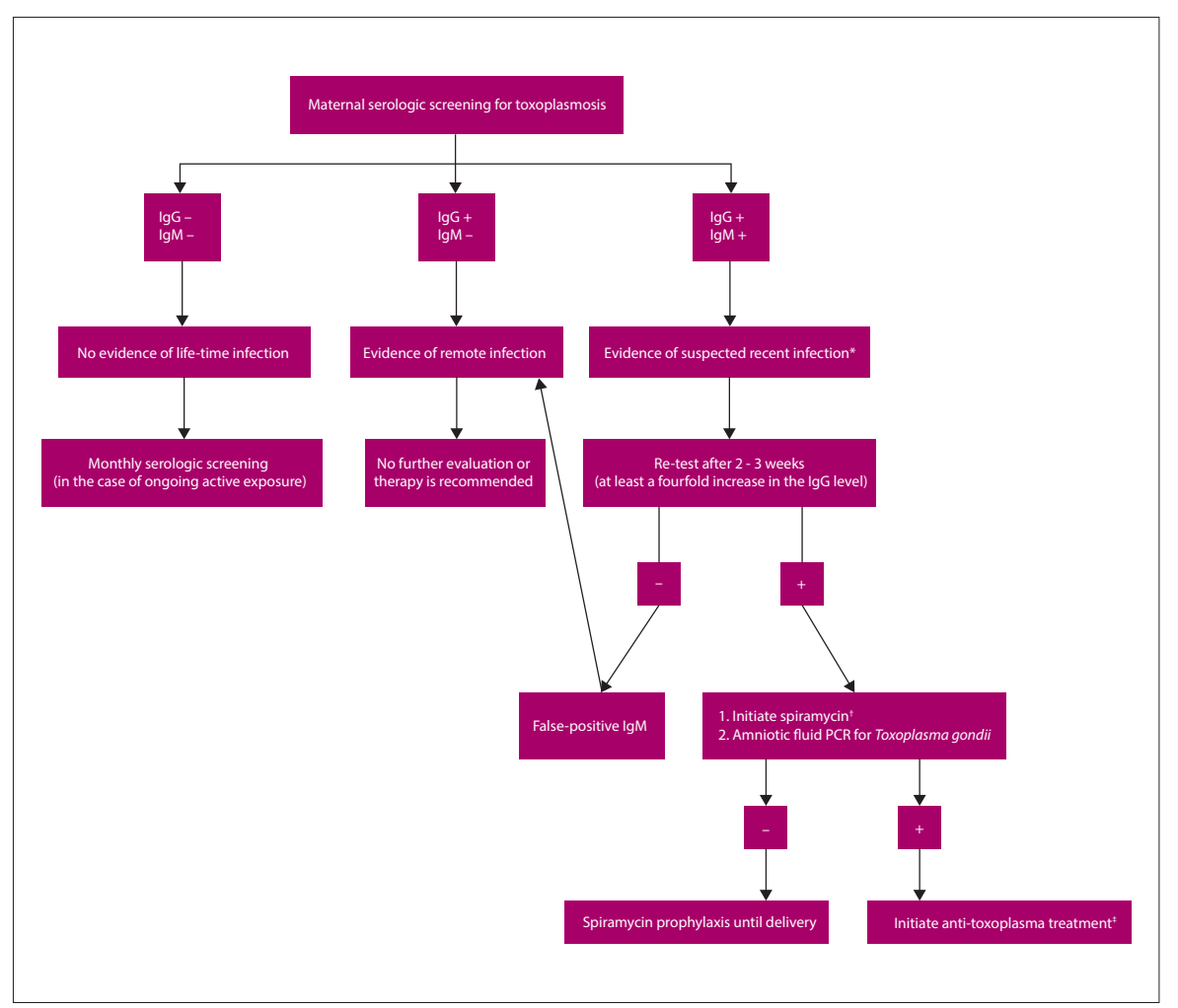

Fig. 1. Recommended algorithm for prenatal screening for congenital toxoplasmosis.

( $I g=$ immunoglobulin; $P C R=$ polymerase chain reaction; $B D=$ twice per day).

${ }^{*}$ A false-positive IgM occurs commonly and a positive result should be confirmed by further evaluation.

${ }^{+}$Spiramycin is generally used if the gestational age is less than 18 weeks.

${ }^{*}$ Anti-toxoplasma treatment with sulfadiazine $(50 \mathrm{mg} / \mathrm{kg} \mathrm{BD}$ ), pyrimethamine ( $2 \mathrm{mg} / \mathrm{kg} /$ day for 2 days, then $1 \mathrm{mg} / \mathrm{kg} 3$ times a week for the rest of the treatment period), and folinic acid (10 mg 3 times a week). The medical treatment should continue for a minimum of 12 months. Anti-toxoplasma treatment is recommended if gestational age is $>18$ weeks. Pyrimethamine should be avoided during the first trimester due to its teratogenic effects. 

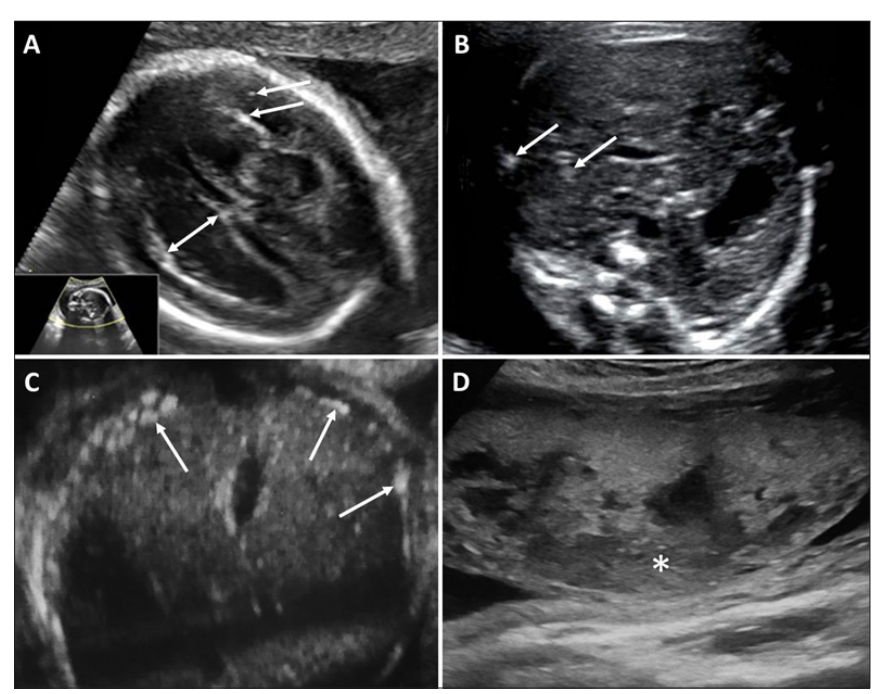

Fig. 2. Possible prenatal ultrasonographic findings in congenital toxoplasmosis (CTX). (A) Head ultrasound of a 23-week fetus with CTX showing lateral ventriculomegaly (bidirectional arrow), as well as periventricular and scattered parenchymal brain echogenic foci representing calcification (arrows); (B) axial abdominal ultrasound of the same fetus demonstrating hepatomegaly and intrahepatic hyperechogenicities (arrows); (C) axial abdominal ultrasound of a 25-week fetus showing multiple echogenic foci on the peritoneal surface representing areas of calcification; (D) an enlarged placenta containing hypoechogenic areas (asterisk) representing placental infarct and intervillous thrombi.

changes (Fig. 2D) such as a thick placenta with hyperechoic and/or necrotic foci are also possible in CTX.

Fetal brain magnetic resonance imaging (MRI) may be used as a complementary study in suspected cases, which can delineate the abnormalities better due its inherent higher soft-tissue resolution in comparison with ultrasound. However, parenchymal calcifications may be undetectable on MRI. ${ }^{[5-8]}$

It should be emphasised that a large proportion of infected fetuses may be completely normal on prenatal ultrasound (57 out of 89 fetuses were normal in a study by Hohlfeld et al. in 1991), but there is still the risk of late complications in these fetuses, which may be prevented partially by initiating early treatment. Therefore, maternal serologic screening is the method of choice that should be offered to pregnant women, and ultrasound may detect only a proportion of more severely affected fetuses. In addition, it has been revealed that the infected fetuses with neuroimaging abnormalities have a poorer prognosis in comparison with those who have normal prenatal neuroimaging results. ${ }^{[9]}$

\section{Mohammad Zare Mehrjardi}

Department of Radiology, Shohada Tajrish Hospital, Shahid Beheshti University of Medical Siences, Tehran, Iran; and Section of Fetal Imaging, Division of Clinical Research, Climax Radiology Education Foundation, Tehran, Iran zare@sbmu.ac.ir

S Afr J Obstet Gynaecol 2018;24(1):4-5. DOI:10.7196/SAJOG.2018. v24i1.1216

1. Jones JL, Lopez A, Wilson M, Schulkin J, Gibbs R. Congenital toxoplasmosis: A review. Obstet Gynecol Surv 2001;56:296-305.

2. Jones J, Lopez A, Wilson M. Congenital toxoplasmosis. Am Fam Physician 2003;67:2131-2138. 3. Kieffer F, Wallon M. Congenital toxoplasmosis. Handb Clin Neurol 2013;112:1099-1101. https:// doi.org/10.1016/B978-0-444-52910-7.00028-3

4. Hampton MM. Congenital toxoplasmosis: A review. Neonatal Netw 2015;34:274-278. https://doi. org/10.1891/0730-0832.34.5.274

5. Malinger G, Werner H, Rodriguez Leonel JC, et al. Prenatal brain imaging in congenital toxoplasmosis. Prenat Diagn 2011;31:881-886. https://doi.org/10.1002/pd.2795

6. Antsaklis A, Daskalakis G, Papantoniou N, Mentis A, Michalas S. Prenatal diagnosis of congenital toxoplasmosis. Prenat Diagnosis 2002;22:1107-1111.

7. Zare Mehrjardi M, Keshavarz E, Poretti A, Hazin AN. Neuroimaging findings of Zika virus infection: A review article. Jap J Radiol 2016;34:765-770. https://doi.org/10.1007/s11604-0160588-5

8. Villena I, Bory JP, Chemla C, Hornoy P, Pinon JM. Congenital toxoplasmosis: Necessity of clinical and ultrasound follow-up despite negative amniocentesis. Prenat Diagnosis 2003;23:1098-1069. https://doi.org/doi:10.1002/pd.754

9. Hohlfeld P, MacAleese J, Capella-Pavlovski M, et al. Fetal toxoplasmosis: Ultrasonographic signs. Ultrasound Obstet Gynecol 1991;1:241-244. https//doi.org/10.1046/j.1469. 0705.1991.01040241.x

Accepted 17 April 2018 